\title{
Avoiding kidney injury in the surgical patient: Further defining the role of age
}

\author{
Natasha Afonso, MD, MPH, and Paul A. Checchia, MD, FCCM, FACC
}

\author{
From the Section of Critical Care Medicine, Texas Children's Hospital, Houston, Tex. \\ Disclosures: Authors have nothing to disclose with regard to commercial support. \\ Received for publication Sept 7, 2017; accepted for publication Sept 8, 2017; available ahead of print Oct 5, 2017. \\ Address for reprints: Paul A. Checchia, MD, FCCM, FACC, Section of Critical Care Medicine, Texas Children's \\ Hospital, 6621 Fannin St, Suite W6006, Houston, TX 77030 (E-mail: checchia@bcm.edu or pachecch@ \\ texaschildrens.org). \\ J Thorac Cardiovasc Surg 2018;155:266-7 \\ $0022-5223 / \$ 36.00$ \\ Copyright (c) 2017 by The American Association for Thoracic Surgery \\ https://doi.org/10.1016/j.jtcvs.2017.09.032
}

Acute kidney injury (AKI) is a common occurrence associated with poor outcomes and increased morbidity and mortality. ${ }^{1}$ The etiology of cardiac surgery-related AKI (CS-AKI) is multifactorial, the result of hemodynamic alterations in renal blood flow, ischemia-reperfusion injury, inflammatory changes, and hemolysis in the setting of diminished autoregulation. ${ }^{2}$ Avoiding CS-AKI has proved challenging because of the difficulty in targeting single pathways in the complex pathophysiology of AKI. ${ }^{3}$ In addition, the exact role of age has yet to be determined. Although it is clear that children have a distinct set of risk factors that differs from the set in the adult population, the subtleties associated with specific age groups have not been investigated.

In this issue of the Journal, Saydy and colleagues ${ }^{4}$ explore the role of age for adults in the risk profile for CS-AKI. Most methods currently used to diagnose AKI (eg, RIFLE, AKIN, and KDIGO criteria) are all based on absolute or percentage changes in serum creatinine. Creatinine varies widely with age, sex, muscle mass, and hydration status, however, and it may not even demonstrate a change until a significant amount of kidney function has already been lost. Early identification of patients at risk for kidney injury is therefore valuable, and the research of Saydy and colleagues ${ }^{4}$ further elucidates patients with risk factors for CS-AKI. Diabetes is a risk factor for CSAKI in the younger age group, whereas smoking and hypertension are risk factors for the older patient group. Across all age groups, high body mass index, coronary artery disease, peripheral vascular disease, anemia, the use of preoperative diuretics, preexisting kidney disease, intra-aortic balloon pump insertion, longer bypass times, hemofiltration, and emergency operative status are additional risk factors for postoperative CS-AKI. This research represents an important contribution to the identification and stratification of patients with AKI after cardiac surgery, notably by examining risk factors across a more defined age range rather than the extremes of child versus adult.

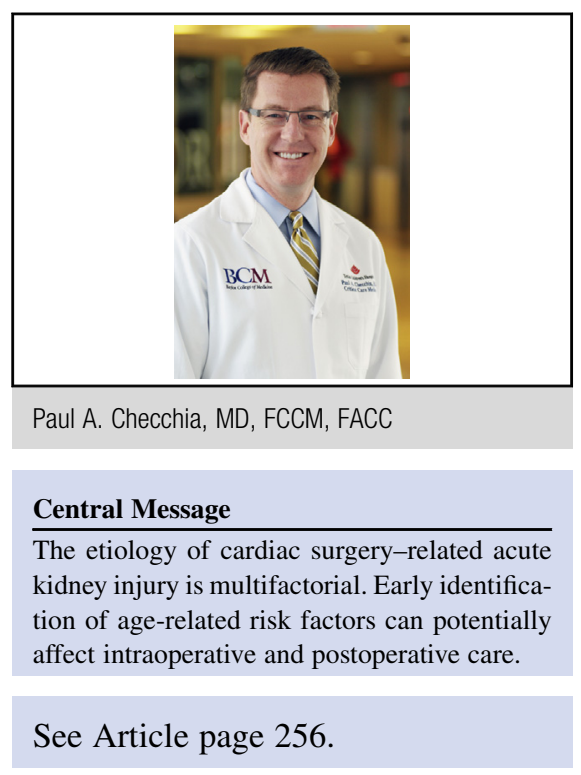

Research dedicated to the early recognition of AKI with urinary and serum biomarkers is underway; however, more studies are required before the use of such markers becomes part of standard practice. In the interim, we must continue to be mindful of the perioperative factors that contribute to kidney damage, especially those factors affected by age. Although there is no singular therapy for mitigating the deleterious effects associated with CS-AKI, this study helps us to consider each patient and individualize therapies according to these factors.

The unremitting progression of time and age is a bond that ties us all. Defining how that progression affects disease and complication rates is an important frontier. As pediatricians, we have always understood the subtle, and not so subtle, impact of age on cardiac surgical risk as we care for a wide age spectrum from the neonate to the adolescent. The research by Saydy and colleagues ${ }^{4}$ introduces the same concept to the adult world. There is a very clichéd answer to the question, "What is old?": "Ten years older than my current age." Alas, when it comes to CS-AKI, that difference may have an impact.

\section{References}

1. Chertow GM, Levy EM, Hammermeister KE, Grover F, Daley J. Independent association between acute renal failure and mortality following cardiac surgery. Am J Med. 1998;104:343-8.

2. Rosner MH, Portilla D, Okusa MD. Cardiac surgery as a cause of acute kidney injury: pathogenesis and potential therapies. J Intensive Care Med. 2008; 23:3-18. 
3. Park M, Coca SG, Nigwekar SU, Garg AX, Garwood S, Parikh CR. Prevention and treatment of acute kidney injury in patients undergoing cardiac surgery: a systematic review. Am J Nephrol. 2010;31: 408-18.
4. Saydy N, Mazine A, Stevens LM, Jeamart H, Demers P, Pagé P, et al. Differences and similarities in risk factors for postoperative acute kidney injury between younger and older adults undergoing cardiac surgery. J Thorac Cardiovasc Surg. 2018;155:256-65. 